\title{
Analysis of the Structural Characteristics of the Online Social Network of Chinese Professional Athletes
}

\author{
Yue Wang $\mathbb{D}^{1},{ }^{1}$ Qian Huang $\mathbb{D}^{1},{ }^{1}$ Qiurong Wang $\mathbb{D}^{1},{ }^{1}$ Yang Xun $\mathbb{D}^{2},{ }^{2}$ Yujiao Tan ${ }^{1},^{1}$ \\ Shuqin Cui $\mathbb{D}^{3},{ }^{3}$ Linxiao Ma, ${ }^{3}$ Penglin Huang $\mathbb{D},{ }^{4}$ Meijuan Cao $\mathbb{D}^{1},{ }^{1}$ and Bin Zhang $\mathbb{D}^{1}$ \\ ${ }^{1} X i$ 'an Physical Education University, Xi'an 710065, China \\ ${ }^{2}$ School of Economics and Finance, Xi'an Jiaotong University, Xi'an 710049, China \\ ${ }^{3}$ Sport College, Xi'an University of Architecture and Technology, Xi'an 710311, China \\ ${ }^{4}$ Shandong Sport University, Jinan 250063, China \\ Correspondence should be addressed to Qian Huang; huangqian168@126.com
}

Received 9 October 2020; Revised 16 January 2021; Accepted 8 February 2021; Published 19 February 2021

Academic Editor: Hongshu Chen

Copyright (C) 2021 Yue Wang et al. This is an open access article distributed under the Creative Commons Attribution License, which permits unrestricted use, distribution, and reproduction in any medium, provided the original work is properly cited.

In response to the lack of research on the online social network structure of athletes, elements of research on the online social network structure of athletes were constructed based on the whole network perspective and through the study of the characteristics of the whole online social network structure of athletes, in order to provide reference for the physical and mental health development of athletes from a new perspective. Data were collected through questionnaires, and several software programs were used to preprocess and analyse the collected data. Through the analysis of the online whole network structure, it was found that the network density of the online support network was generally greater than that of the online discussion network, and athletes still showed stronger practical support demands and behaved more rationally in the process of training and learning life, while from the perspective of the relationship structure, the athletes' family and classmates' online support is weaker than that in previous studies; in terms of the whole network, strong relationships still dominate in this population, while attention should be paid to the impact of weak relationships.

\section{Introduction}

China's competitive sports implement the "one piece of mind," "one organization," and "consistent training" training system, and athletes are in a relatively independent and closed environment for a long time. The training, learning, and living environment of athletes is relatively independent and closed for a long time, and the breadth, depth, and integration of real social interaction are subject to certain limitations [1-5]. With the rapid development of the Internet, online social network platforms such as WeChat, Weibo, QQ, and social networking sites have been integrated into people's daily lives, and the huge Internet user base has formed an intricate virtual society-Online Social Network
(OSN) [6-14], which provides athletes with a more modern and diverse way to socialize [15-17].

Athletes, in particular Chinese competitive athletes, have very specific social relationships. The offline social relationships of athletes described in this article are relatively closed, i.e., closed for a certain amount of time and closed for a certain amount of space. The reality is that athletes training in preparation for an event, for example, will remain in one or several relatively closed environments for a period of time (e.g., weeks or even months), and connections to the outside world are negligible compared to the large number of internal connections. Due to the influence of offline social interaction, this group is also relatively closed online compared to the general public. 
Online social networks are having an increasing impact on athletes' social support and social inclusion, as athletes learn about society, access information, express their aspirations, and have greater emotional resonance through the Internet [18].

Due to the special nature of athletes' social relationships, online social networking has become an important way for athletes to connect with others. The survey data shows that athletes' social networks are limited in time and space compared to those of the general public, in that their online time is relatively limited and fixed, and their online activities are relatively concentrated. The challenge in studying this online social network of this population is that online data is not easy to collect and there is a lack of references, so we continue to use traditional questionnaires. The innovation of this paper is to conduct an online social support network study based on the previous offline social support network, which is an exploratory approach to fill the gap in this field in China, and is groundbreaking in terms of both the athlete population and the research methodology.

Current research on athletes' social networks focuses on the attribute variables and structural analysis of real social networks, and their online social data and research are lacking. Athletes' online social network is an open and complex system, a large-scale collection consisting of connected relationships between athletes and other network citizens, including teammates, coaches, family members, hometown, friends, and even strangers [19, 20]. Thinking about and understanding athletes' online social structure from different dimensions and studying the inner laws of professional athletes' network behaviour at multiple levels (micro-, meso-, and macrolevel) can help grasp the mechanisms of athletes' behavioural patterns and provide references for improving their online social support networks, promoting social integration, and further improving the sports system.

Online social networks are an extension of social networks $[21,22]$, a form of social networks existing in another space, and their structural characteristics can be analysed by applying the methods of social network analysis. According to the different boundaries of social networks, the study of social network structure is divided into two directions: individual networks and whole networks [23, 24]. Individual networks locate the network with individuals as the centre and the individual characteristics and behavioural concepts of concern, while whole networks consist of a set of specific individuals and the interrelationships between them, and the network members have relatively obvious boundaries [25-27]. The network as a whole exhibits a certain structure and has an impact on the actors within it [28]. In other words, the "structuralist" perspective of "social structure" influences the "dynamic role." This study examines the structural characteristics of athletes' whole online social network by collecting online data from athletes in order to provide a new perspective on athletes' physical and mental health development.

\section{Elements of Athletes' Online Social Structure}

The term "online social network" refers to the network of social users in the Internet [29]. According to the EU study on social computing, online social networks can be divided into four categories: instant messaging (WeChat, QQ, etc.), online social networking (Facebook, Renren, etc.), microblogging (Twitter, Weibo, etc.), and shared space (forums, blogs, etc.). The latest data released by CNNIC shows that applications such as WeChat, short video, and live streaming are increasingly integrated with the lives of Chinese Internet users. In the preliminary stage of this study, a sample of 451 athletes were surveyed on online social network applications, and the most frequently used application by respondents was instant messaging, accounting for $93.1 \%$, of which WeChat had the highest usage rate.

As shown in Figure 1, online social network for athletes consists of two categories: social support network and social discussion network [23, 30]. The online social support network is based on the Van der Poole social support classification method, and it is divided into social online support, emotional online support, achievement online support, and practical online support. Based on the Institute of Population and Development of Xi'an Jiaotong University's social network of migrant workers and the social network of athletes under Huang Qian's complex network, the online social discussion network is analysed [23, 31] from four aspects: employment online discussion, revenue online discussion, professional online discussion, and love and marriage online discussion. The questionnaire data are collected by positioning method.

\section{Data Collection}

Whole social networks require closed groups, and such sociologically significant networks are generally not large in size. This study sampled a closed group formed by 147 professional taekwondo athletes from the Shaanxi Boxing and Taekwondo Sports Management Centre. The questionnaire was divided into two parts, the first part investigated the basic information of the athletes, including gender, date of birth, and total number of WeChat friends, and the second part designed a list of 147 athletes' names and code names according to the roster method [32, 33].

The data collection was conducted from January 10 to January 13, 2020. To ensure accurate and valid data, the author interpreted the questions on site, and in the second part of the questionnaire filling, athletes first filled in their own code names according to the code name list and then selected other athletes who had correspondence with them in the test questions item by item and filled in their corresponding code names. Through a survey of coaches and relevant researchers and drawing on Huang Qian et al.'s analysis of athletes' whole social networks [3, 4], the following measurement questions about athletes' online social networks were selected: (1) Who do you interact with on 


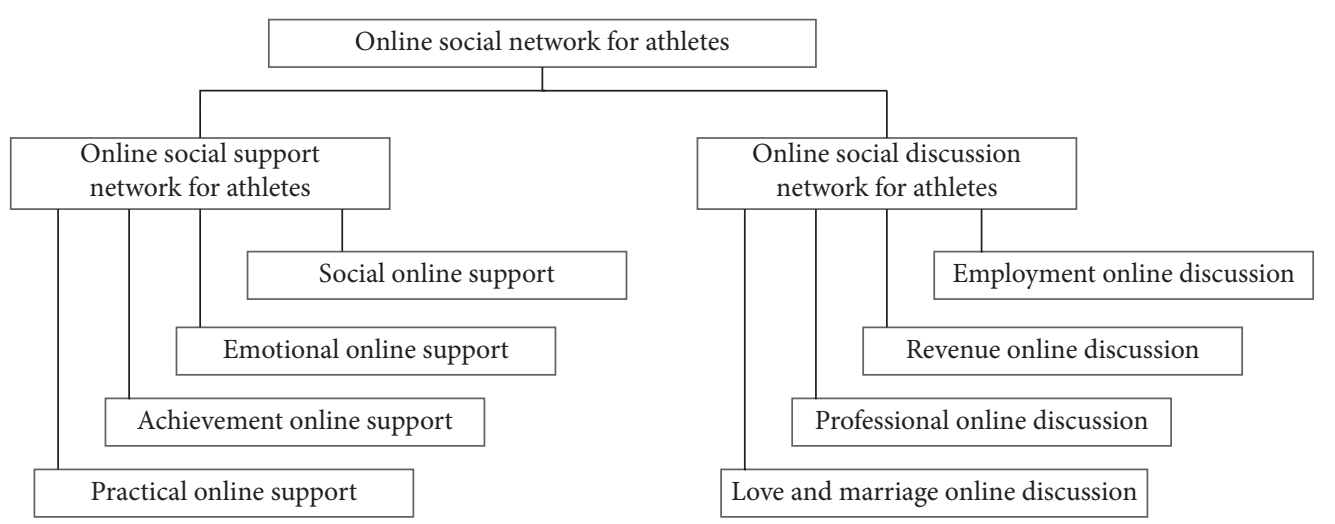

FIgURE 1: Organization structure of social network of athletes.

online platforms (conversations, holiday greetings, file transfers, etc.)? (2) In online chats, to whom do you confide your feelings? (3) Who do you turn to for help with small daily tasks on online platforms (including online advice, online payments)? (4) Who are the people who push information online (private messages, group notifications, or sharing with friends) that would be helpful to your training? (5) Who would you like to talk to online about issues related to training or sports team management, etc.? (6) Who would you like to discuss marriage topics with online? (7) Who would you like to discuss employment issues with online? (8) With whom would you like to discuss issues such as income online? Respondents ticked each item according to the list, and the relationship was recorded as " 1 " if selected and " 0 " otherwise. Eight relationship matrices consisting of " 0 " and "1" are formed, for example, in Table 1. The questionnaires were collected on site and four athletes were away for training during the collection period, so the author instructed them to complete the questionnaires online via the Internet. After the questionnaires were collected, the "informants" were used to understand the general situation of the training team, and the questionable data was returned to the author. 147 valid questionnaires were collected. In this paper, the data cleaning and two-party relationship data statistics are done by self-programmed Excel VBA, while other characteristic index calculation and topology drawing are processed by Ucinet and Gephi software.

To illustrate the relationship matrix for athletes using an online practical support network as an example, it is sufficient to intercept parts of the matrix that illustrate the problem, as it is large. In Table $1, N$ represents the total number of nodes and ai $(i=1,2, \ldots 20)$ denote athlete nodes, and due to space limitations, only a1 to $a 20$ are taken. Using formula (1) to calculate its density which is only 0.0273 , we can find that the matrix is a sparse matrix ( $L$ represents the actual number of connections; $N=147$ is the number of nodes; generally less than 0.05 is considered as a sparse matrix), in line with the basic characteristics of social networks; secondly, the distribution of connections has no obvious regularity (i.e., nondiagonal array, triangular array, unit array, etc.), which is a random network. Other thematic networks $B \sim H$ are similar.

$$
d(G)=\frac{L}{N(N-1)} .
$$

\section{Analysis of the Characteristics of Whole Online Social Network}

4.1. Topology of Athletes Network. In order to visualise the relationship structure of the eight online social networks for athletes, the online social network topology diagram [34-36] was drawn using Gephi as shown in Figure 2, with each of the eight subgraphs labelled with the letters $A-H$. In each directed graph, the arrow indicates the direction of the relationship; e.g., a23 points to $a 8$ in subgraph $\mathrm{B}$, which means that $x m 23$ is willing to confide in $x m 8$ about what is on his mind. These eight subnetworks are derived from the eight relationship matrices $A$ to $H$ collated through the questionnaire as isomorphic to Table 1 .

As can be seen, the online practical support network is the most densely connected and all nodes are connected to each other, indicating that athletes are most socially active and connected online. The practical online support network is also denser, with no isolated points. The emotional online support, employment online discussion, achievement online support, revenue online discussion, professional online discussion, and love and marriage online discussion networks all have isolated points, especially the love and marriage online discussion networks, which have the sparsest relationships, the most isolated points, and multiple subnetworks that are not connected to each other.

4.2. Network Viscosity. Network viscosity reflects the degree of overall network node association and overall cohesion, and is measured by network density and network distance. 
TABLe 1: Athlete online relationship matrix.

\begin{tabular}{ccccccccccccccccccccccccc}
\hline$N$ & $a 1$ & $a 2$ & $a 3$ & $a 4$ & $a 5$ & $a 6$ & $a 7$ & $a 8$ & $a 9$ & $a 10$ & $a 11$ & $a 12$ & $a 13$ & $a 14$ & $a 15$ & $a 16$ & $a 17$ & $a 18$ & $a 19$ & $a 20$ & $\ldots$ \\
\hline$a 1$ & 0 & 1 & 1 & 0 & 0 & 0 & 0 & 1 & 0 & 0 & 0 & 0 & 0 & 0 & 0 & 0 & 0 & 0 & 0 & 0 & $\ldots$ \\
$a 2$ & 1 & 0 & 1 & 0 & 0 & 0 & 0 & 0 & 0 & 0 & 0 & 0 & 0 & 0 & 0 & 0 & 0 & 0 & 0 & 0 & $\ldots$ \\
$a 3$ & 1 & 1 & 0 & 0 & 1 & 0 & 0 & 0 & 0 & 0 & 0 & 0 & 0 & 0 & 0 & 0 & 0 & 0 & 0 & 0 & $\ldots$ \\
$a 4$ & 0 & 0 & 0 & 0 & 0 & 1 & 0 & 0 & 1 & 0 & 0 & 0 & 0 & 0 & 0 & 0 & 0 & 0 & 0 & 0 & $\ldots$ \\
$a 5$ & 0 & 0 & 0 & 0 & 0 & 0 & 0 & 1 & 0 & 0 & 0 & 0 & 0 & 0 & 0 & 0 & 0 & 0 & 0 & 0 & $\ldots$ \\
$a 6$ & 0 & 0 & 0 & 0 & 0 & 0 & 0 & 0 & 0 & 0 & 0 & 0 & 0 & 0 & 0 & 0 & 0 & 0 & 0 & 0 & $\ldots$ \\
$a 7$ & 0 & 0 & 0 & 0 & 0 & 0 & 0 & 1 & 0 & 0 & 0 & 0 & 0 & 0 & 0 & 0 & 0 & 0 & 0 & 0 & $\ldots$ \\
$a 8$ & 1 & 0 & 0 & 0 & 1 & 0 & 0 & 0 & 0 & 0 & 0 & 0 & 0 & 0 & 0 & 0 & 0 & 0 & 0 & 0 & $\ldots$ \\
$a 9$ & 0 & 0 & 0 & 1 & 0 & 1 & 1 & 0 & 0 & 0 & 0 & 0 & 0 & 0 & 0 & 0 & 0 & 0 & 0 & 0 & $\ldots$ \\
$a 10$ & 0 & 0 & 0 & 0 & 1 & 0 & 0 & 0 & 0 & 0 & 0 & 0 & 0 & 0 & 0 & 0 & 0 & 0 & 0 & 0 & $\ldots$ \\
$a 11$ & 0 & 0 & 0 & 0 & 0 & 0 & 0 & 0 & 0 & 0 & 0 & 0 & 0 & 0 & 0 & 0 & 0 & 0 & 0 & 0 & $\ldots$ \\
$a 12$ & 0 & 0 & 0 & 0 & 0 & 0 & 0 & 0 & 0 & 0 & 1 & 0 & 0 & 0 & 0 & 0 & 0 & 0 & 0 & 0 & $\ldots$ \\
$a 13$ & 0 & 0 & 0 & 0 & 0 & 0 & 0 & 0 & 0 & 0 & 0 & 0 & 0 & 0 & 0 & 0 & 0 & 0 & 0 & 0 & $\ldots$ \\
$a 14$ & 0 & 0 & 0 & 0 & 0 & 0 & 0 & 0 & 0 & 0 & 0 & 0 & 0 & 0 & 1 & 0 & 0 & 0 & 0 & 0 & $\ldots$ \\
$a 15$ & 0 & 0 & 0 & 0 & 0 & 0 & 0 & 0 & 0 & 0 & 0 & 1 & 0 & 1 & 0 & 0 & 0 & 0 & 0 & 1 & $\ldots$ \\
$a 16$ & 0 & 0 & 0 & 0 & 0 & 0 & 0 & 0 & 0 & 0 & 0 & 0 & 0 & 0 & 0 & 0 & 0 & 0 & 1 & 0 & $\ldots$ \\
$a 17$ & 0 & 0 & 0 & 0 & 0 & 0 & 0 & 0 & 0 & 0 & 1 & 1 & 0 & 0 & 1 & 1 & 0 & 1 & 0 & 1 & $\ldots$ \\
$a 18$ & 0 & 0 & 0 & 0 & 1 & 0 & 0 & 0 & 1 & 0 & 1 & 1 & 0 & 0 & 1 & 0 & 1 & 0 & 1 & 1 & $\ldots$ \\
$a 19$ & 0 & 0 & 0 & 0 & 0 & 0 & 0 & 0 & 0 & 0 & 0 & 1 & 0 & 0 & 0 & 1 & 1 & 1 & 0 & 1 & $\ldots$ \\
$a 20$ & 0 & 0 & 0 & 0 & 0 & 0 & 0 & 0 & 0 & 0 & 0 & 0 & 0 & 0 & 1 & 0 & 0 & 0 & 0 & 0 & $\ldots$ \\
$\ldots$ & $\ldots$ & $\ldots$ & $\ldots$ & $\ldots$ & $\ldots$ & $\ldots$ & $\ldots$ & $\ldots$ & $\ldots$ & $\ldots$ & $\ldots$ & $\ldots$ & $\ldots$ & $\ldots$ & $\ldots$ & $\ldots$ & $\ldots$ & $\ldots$ & $\ldots$ & $\ldots$ & $\ldots$ \\
\hline
\end{tabular}

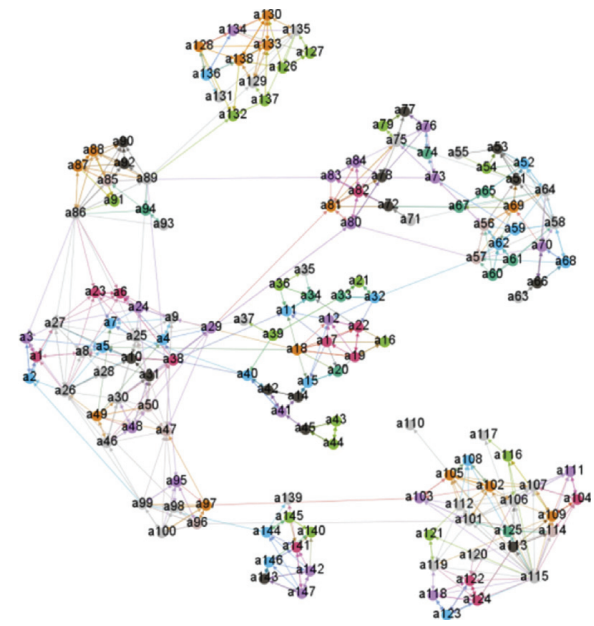

(a)

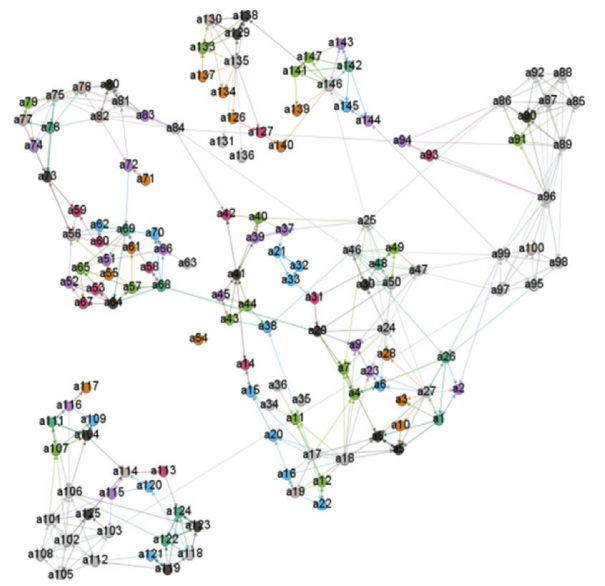

(c)

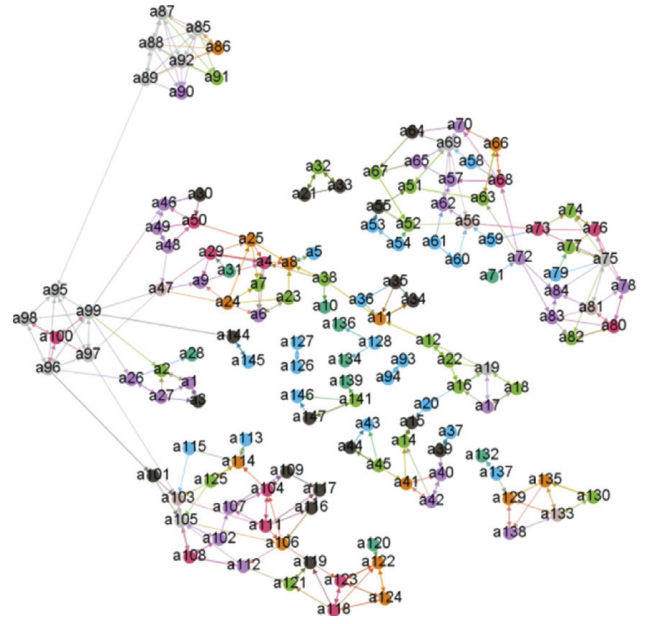

(b)

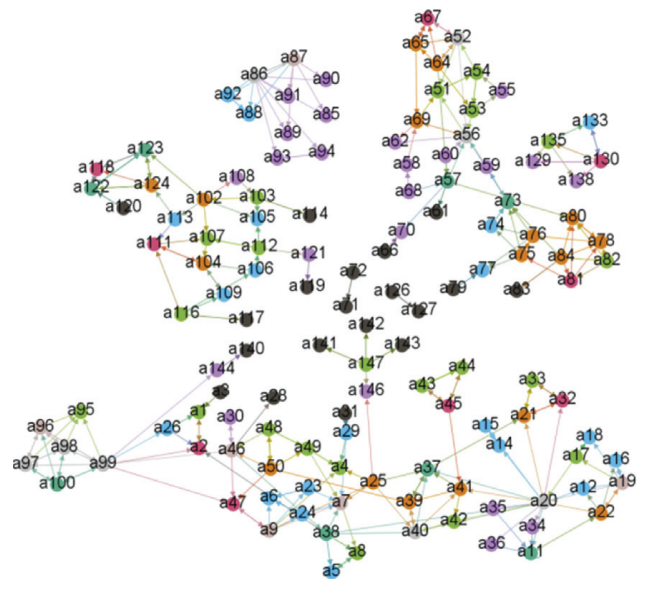

(d)

FIgURE 2: Continued. 


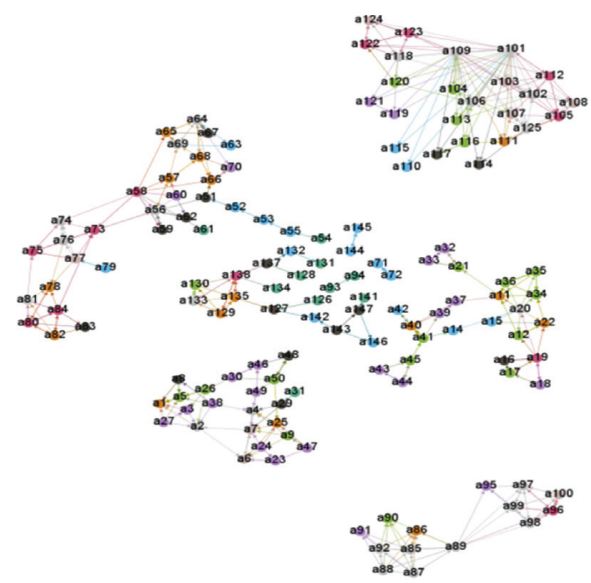

(e)

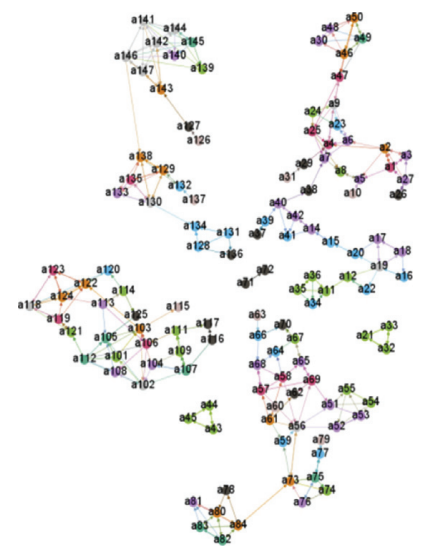

(g)

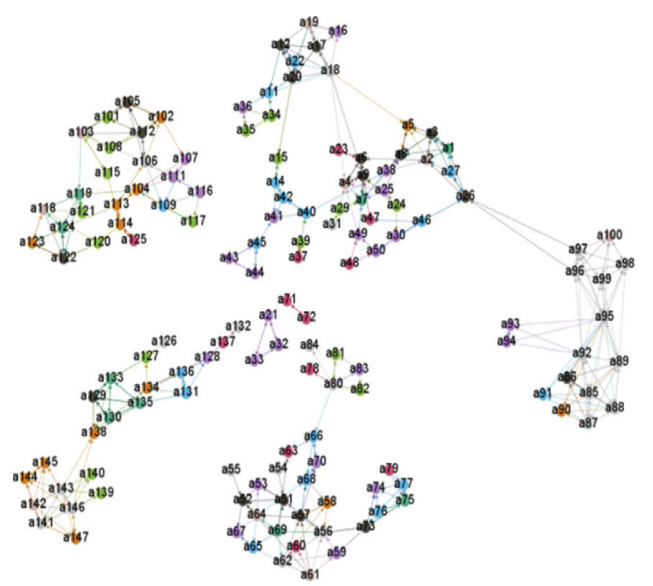

(f)

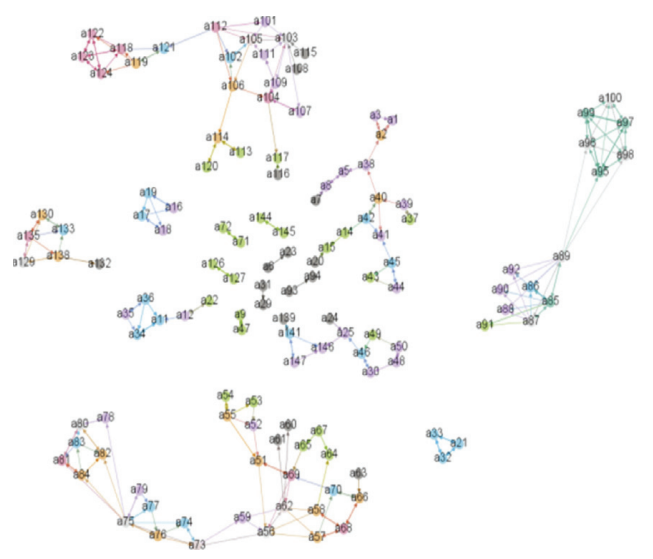

(h)

Figure 2: Topology of the online social network. (a) Practical online support network. (b) Emotional online support network. (c) Social online support network. (d) Employment online discussion network. (e) Achievement online support network. (f) Revenue online discussion network. (g) Professional online discussion network. (h) Love and marriage online discussion network.

Network density is an important metric in the structural form of a network and is defined as the ratio of the number of edges actually present in the network to the maximum number of edges that can be accommodated. Network density is commonly used in online social networks to measure the density of social relationships, with higher density indicating more connections and the greater effectiveness of the network's influence on nodes. The network distance is used to describe the length of the shortest path between nodes, and the clustering coefficient reflects the degree of network aggregation. The combination of network distance and clustering coefficient can demonstrate the "small world" effect, showing how nodes are embedded in their surrounding nodes. By referring to relevant research results [37-39], an indicator is proposed here to combine the two, as shown in (2), and the last column of $C d$ is obtained as in Table 2; the higher the $C d$, the more obvious the "small world" effect.

$$
C d_{i}=\frac{\left(c i / \sum_{i} c i+d i / \sum_{i} d_{i}\right)}{2} .
$$

Statistics on the whole network viscosity characteristics of the athletes' online social networks are shown in Table 2. The network density of each of the athletes' online social networks was in the interval $[0.016,0.028]$, indicating that the network had essentially equal influence on the athletes. Among the online social support networks, emotional online support was the most lacking, and their network density was only 0.016; among the online social discussion networks, the athletes were more concerned with income issues. Comparing the average distance of the networks revealed that athletes had the longest chain of online practical support relationships and were prone to extend online practical support relationships. The average clustering coefficient for love and marriage online discussions was only 0.3 , with the 
TABLE 2: The viscosity characteristics of online social network.

\begin{tabular}{lccc}
\hline Name of network & Density & Mean distance & Average clustering coefficient \\
\hline Practical online support network & 0.028 & 5.663 & 0.401 \\
Emotional online support network & 0.017 & 4.025 & 0.310 \\
Social online support network & 0.024 & 4.672 & 0.361 \\
Employment online discussion network & 0.016 & 3.138 & 0.314 \\
Achievement online support network & 0.02 & 2.781 & 0.379 \\
Revenue online discussion network & 0.021 & 3.059 & 0.388 \\
Professional online discussion network & 0.019 & 2.911 & 0.388 \\
Love and marriage online discussion network & 0.016 & 2.967 & 0.3 \\
\hline
\end{tabular}

sparsest network relationships. The "small world" effect of practical online support network is most evident in the $C d$ measure.

4.3. Centrality. Centrality measures the structural properties of social networks at a microlevel and is usually measured by three indicators: degree centrality, closeness centrality, and betweenness centrality.

As shown in (3), $g$ represents the size of the network and degree centrality $C_{D}$ is the most direct indicator of the centrality of a network node; it measures the total number of direct links between a node and other nodes. The higher the degree centrality is, the more important the node is in the network; in the case of directed graphs, the two parameters out-degree and in-degree need to be considered.

$$
\left\{\begin{array}{l}
C_{D}\left(N_{i}\right)=\sum_{j=1}^{g} x_{i j}, \\
C_{D}^{\prime}\left(N_{i}\right)=\frac{C_{D}\left(N_{i}\right)}{g-1}, \quad i \neq j .
\end{array}\right.
$$

Closeness centrality $C(x)$ examines the extent to which a node propagates information without relying on other nodes; if a node is at a short distance from all other nodes, then that point is at the overall centre. As shown in (4), $d(x, y)$ indicates the distance between node $x$ and node $y$ :

$$
C(x)=\frac{1}{\sum_{y} d(y, x)} .
$$

As shown in (4), betweenness centrality characterises the extent to which a node is known as a bridge to the various others or the extent to which it can control others. $\sigma_{s t}(v)$ denotes the number of shortest paths of two nodes through point $v$, and $\sigma_{s t}$ denotes the total number of shortest paths of two nodes.

$$
C B(v)=\sum_{s \neq v \neq t} \frac{\sigma_{s t}(v)}{\sigma_{s t}} .
$$

The data was processed using the formulae above. The characteristics of the whole online social network structure of the athletes in-degree and out-degree are shown in Table 3 . The maximum values show that the out-degree is generally greater than the in-degree, indicating that athletes actively seek online support and online discussion more than they passively receive it. The minimum value of in-degree and out-degree for all networks is 0 , indicating that there are athletes who do not engage in online support and online discussion behaviours with others in their sport team. In addition, there is more variation between individual athletes, such as the practical online support network and achievement online support networks, which have a maximum value of 25, while both have a minimum value of 0 .

Table 4 shows the parameter values for the whole online social network centrality characteristics of the athletes. Comparing the indicators of eccentricity, closeness centrality, harmonic centrality, and betweenness centrality reveals that athletes have a preference for seeking achievement support over other online support. Online social discussion and online social support in general show a more active and active search for support and discussion, while acceptance is more passive. In terms of the propagation of online social support and online social discussion relationships, athletes were more likely to extend their daily help support relationships and were susceptible to the influence of intermediaries. Overall, the higher closeness centrality of love and marriage online discussion network indicates a higher chance of connection between nodes. Harmonic centrality is usually for nonconnected networks and can be ignored here. Network with higher betweenness centrality indicates that more nodes in the network play a role in connecting these groups together, such as practical online support network.

4.4. Bipartite Relationship, Tripartite Relationship. Bipartite relationship is the basic unit of statistical analysis of social networks. For directed networks, the bipartite relationship between two nodes in the network includes reciprocal relationships, one-way relationships, and nulls.

Tripartite relationships, i.e., relationships between three nodes, are formed by combining three pairs of bipartite relationships. The tripartite structure is the basis of social structure and is described by scholars such as Holland as 16 isomorphic categories; each represented by three or four symbols: the first number represents the number of reciprocal tripartite relations; the second number represents the number of asymmetrical tripartite relations; the third number represents the number of nulls in tripartite 
TABLE 3: Characteristics of the whole online social network structure of athletes.

\begin{tabular}{|c|c|c|c|c|c|c|c|c|}
\hline \multirow{2}{*}{ Name } & \multicolumn{4}{|c|}{ Out-degree } & \multicolumn{4}{|c|}{ In-degree } \\
\hline & Mean & Std. & Max & Min & Mean & Std. & Max & Min \\
\hline Practical online support network & 4.068 & 3.362 & 25.000 & 0.000 & 4.068 & 1.925 & 10.000 & 0.000 \\
\hline Emotional online support network & 2.433 & 1.880 & 10.000 & 0.000 & 2.433 & 1.537 & 6.000 & 0.000 \\
\hline Social online support network & 3.418 & 3.055 & 14.000 & 0.000 & 3.411 & 1.863 & 8.000 & 0.000 \\
\hline Employment online discussion network & 2.092 & 2.238 & 15.000 & 0.000 & 2.092 & 1.444 & 7.000 & 0.000 \\
\hline Achievement online support network & 2.837 & 3.384 & 25.000 & 0.000 & 2.837 & 1.869 & 9.000 & 0.000 \\
\hline Revenue online discussion network & 2.936 & 2.550 & 15.000 & 0.000 & 2.957 & 1.808 & 8.000 & 0.000 \\
\hline Professional online discussion network & 2.716 & 2.358 & 14.000 & 0.000 & 2.752 & 1.657 & 7.000 & 0.000 \\
\hline Love and marriage online discussion network & 2.015 & 1.728 & 9.000 & 0.000 & 2.015 & 1.468 & 8.000 & 0.000 \\
\hline
\end{tabular}

TABle 4: Central characteristics of the whole online social network structure of athletes.

\begin{tabular}{|c|c|c|c|c|}
\hline Name & Eccentricity & Closeness centrality & Harmonic centrality & Betweenness centrality \\
\hline Practical online support network & 8.247 & 0.294 & 0.368 & 251.397 \\
\hline Emotional online support network & 4.376 & 0.435 & 0.502 & 57.780 \\
\hline Social online support network & 5.266 & 0.366 & 0.440 & 129.133 \\
\hline Employment online discussion network & 3.092 & 0.436 & 0.491 & 21.084 \\
\hline Achievement online support network & 2.823 & 0.497 & 0.558 & 17.440 \\
\hline Revenue online discussion network & 3.832 & 0.450 & 0.528 & 28.336 \\
\hline Professional online discussion network & 3.694 & 0.453 & 0.531 & 20.667 \\
\hline Love and marriage online discussion network & 2.718 & 0.550 & 0.607 & 15.389 \\
\hline
\end{tabular}

relations; and if there is a final letter, it is used to distinguish between two similar tripartite relations ( $T$ for transmission, $C$ for circular, $U$ for up, and $D$ for down).

The overall number of relationships between the 147 nodes in this study totalled 3,668, and Tables 5 and 6 show the proportion of each relationship to the overall relationship in the bipartite and tripartite relationships, respectively. It can be seen that the athlete group has an absolute majority proportion of the nulls in all 8 networks. The proportion of one-way relationships is very small, while the proportion of reciprocal relationships is even smaller. This suggests that online one-way communication between groups of athletes is rare and two-way interaction is more difficult. Examining the actual situation, based on the training patterns of the athletes, this is not unrelated to the regulation of communication tools. A comparison of the bipartite relationship data reveals that athletes interact with each other mainly through social online support network to enhance communication with each other, but less in terms of marriage and emotions, which may be that athletes are close to each other before they engage in emotional confessions and discussions on topics such as marriage.

The very large percentage of 003 in the athletes' tripartite relationships suggests that in most cases there are no online support and online discussion relationships between two out of three people in the athletes' group. 012 had the second highest proportion, indicating that a larger proportion of the online relationships among the three athletes, of which only two had relationships with each other, were difficult to transmit among the three and that it was difficult to communicate and collide their perceptions effectively. 300 and 030C had the smallest proportion of both, indicating that a small proportion of the three athletes had one-way transmission relationships and two-way transmission relationships; i.e., the whole online social network of athletes has a poor sense of identity and belonging in the group. Bipartite relationships are the simplest relationships to consider and tripartite relationships have complex social relationship attributes and so are generally of greater concern.

4.5. Community Structure. Realistic social networks are often not evenly distributed, but consist of many subnetworks with high similarity of nodes within the subnetwork and low similarity of nodes outside the subnetwork, resulting in a community structure. The modularity $Q$ is used to quantify or judge the merit of community division in a network, as shown in (6). It is defined as the ratio of the total number of edges within the community to the total number of edges in the network minus an expectation value that is the magnitude of the ratio of the total number of edges within the community to the total number of edges in the network that would result from the same community assignment if the network was set as a random network. If $Q$ is greater than 0.3 , then the network has a significant community structure [40].

$$
Q=\sum_{i}\left(e_{i i}-a_{i}^{2}\right)=\sum_{i} e_{i i}-\sum_{i} a_{i}^{2}=\text { Tre }-\left\|e^{2}\right\| .
$$

Figure 3 shows the modularity of the online social support network and the online social discussion network. It can be seen that the modularity $Q$ of both is even more than 0.8 . It indicates that community structure exists widely in 
TABLE 5: Distribution of bipartite relationship.

\begin{tabular}{lccc}
\hline Name & Reciprocal relationships (\%) & One-way relationship (\%) & Nothingness (\%) \\
\hline Practical online support network & 3.05 & 6.69 & 90.26 \\
Emotional online support network & 0.49 & 2.49 & 97.02 \\
Social online support network & 1.42 & 3.93 & 94.65 \\
Employment online discussion network & 0.24 & 3.61 & 96.14 \\
Achievement online support network & 0.59 & 4.08 & 95.34 \\
Revenue online discussion network & 0.22 & 1.39 & 98.39 \\
Professional online discussion network & 0.51 & 4.06 & 95.43 \\
Love and marriage online discussion network & 0.33 & 3.31 & 96.36 \\
\hline
\end{tabular}

TABle 6: Distribution of tripartite relationship.

\begin{tabular}{|c|c|c|c|c|c|c|c|c|}
\hline Type & $\begin{array}{l}\text { Practical } \\
\text { online } \\
\text { support } \\
\text { network }\end{array}$ & $\begin{array}{l}\text { Emotional } \\
\text { online } \\
\text { support } \\
\text { network }\end{array}$ & $\begin{array}{l}\text { Social } \\
\text { online } \\
\text { support } \\
\text { network }\end{array}$ & $\begin{array}{c}\text { Employment } \\
\text { online discussion } \\
\text { network }\end{array}$ & $\begin{array}{l}\text { Achievement } \\
\text { online support } \\
\text { network }\end{array}$ & $\begin{array}{l}\text { Revenue } \\
\text { online } \\
\text { discussion } \\
\text { network }\end{array}$ & $\begin{array}{l}\text { Professional } \\
\text { online } \\
\text { discussion } \\
\text { network }\end{array}$ & $\begin{array}{c}\text { Love } \\
\text { and marriage } \\
\text { online } \\
\text { discussion } \\
\text { network } \\
\end{array}$ \\
\hline 003 & 73.5 & 91.32 & 84.74 & 89.04 & 86.79 & 95.24 & 87.84 & 92.62 \\
\hline 012 & 16.47 & 7.05 & 10.66 & 9.72 & 10.94 & 4.06 & 9.67 & 6.02 \\
\hline 102 & 7.72 & 1.39 & 3.89 & 0.67 & 1.58 & 0.63 & 1.87 & 1.12 \\
\hline 021D & 0.41 & 0.05 & 0.14 & 0.20 & 0.23 & 0.02 & 0.17 & 0.08 \\
\hline $021 \mathrm{U}$ & 0.27 & 0.06 & 0.10 & 0.17 & 0.11 & 0.01 & 0.19 & 0.06 \\
\hline $021 \mathrm{C}$ & 0.36 & 0.07 & 0.14 & 0.10 & 0.15 & 0.02 & 0.10 & 0.05 \\
\hline $111 \mathrm{D}$ & 0.32 & 0.02 & 0.13 & 0.02 & 0.03 & 0.01 & 0.03 & 0.04 \\
\hline $111 \mathrm{U}$ & 0.40 & 0.01 & 0.09 & 0.03 & 0.08 & 0.01 & 0.05 & 0.00 \\
\hline $030 \mathrm{~T}$ & 0.10 & 0.01 & 0.03 & 0.04 & 0.04 & 0.00 & 0.03 & 0.00 \\
\hline 030C & 0.01 & 0.00 & 0.00 & 0.00 & 0.00 & 0.00 & 0.00 & 0.01 \\
\hline 201 & 0.12 & 0.01 & 0.03 & 0.00 & 0.01 & 0.00 & 0.02 & 0.00 \\
\hline $120 \mathrm{D}$ & 0.08 & 0.00 & 0.02 & 0.00 & 0.01 & 0.00 & 0.01 & 0.00 \\
\hline $120 \mathrm{U}$ & 0.06 & 0.00 & 0.01 & 0.00 & 0.02 & 0.00 & 0.01 & 0.00 \\
\hline $120 \mathrm{C}$ & 0.05 & 0.00 & 0.01 & 0.00 & 0.00 & 0.00 & 0.00 & 0.00 \\
\hline 210 & 0.10 & 0.00 & 0.01 & 0.00 & 0.01 & 0.00 & 0.01 & 0.00 \\
\hline 300 & 0.03 & 0.00 & 0.01 & 0.00 & 0.00 & 0.00 & 0.00 & 0.00 \\
\hline
\end{tabular}

Modularity: 0.854

Modularity with resolution: 0.854

Number of communities: 13

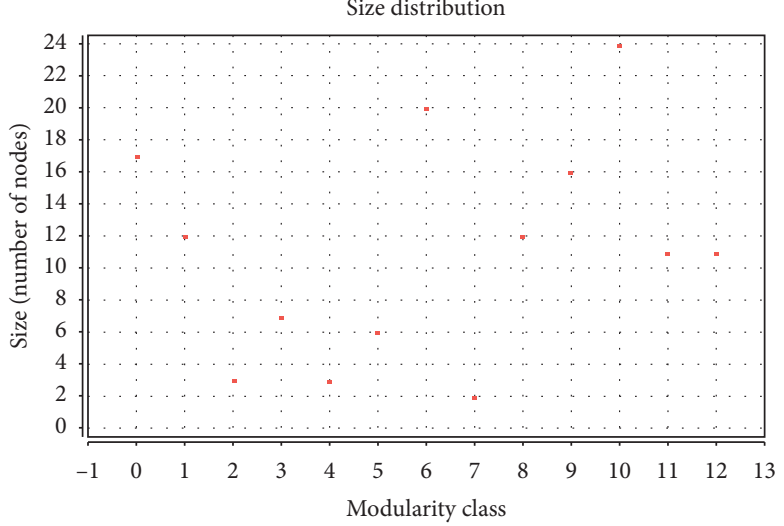

(a)
Modularity: 0.867

Modularity with resolution: 0.867

Number of communities: 19

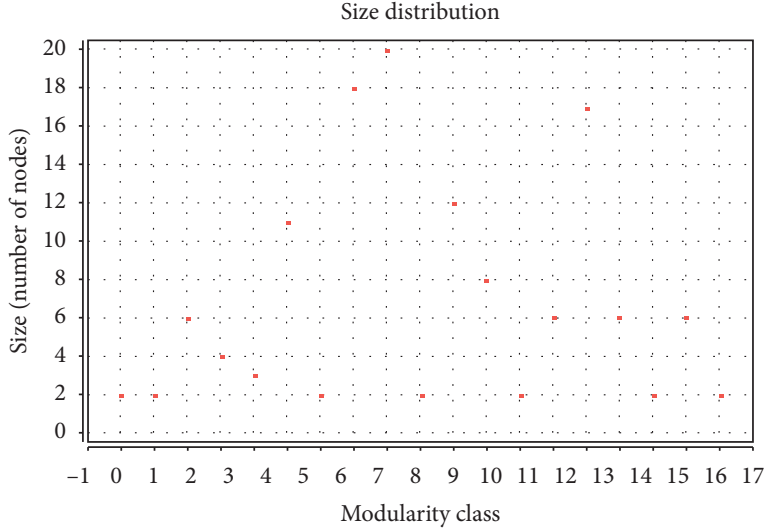

(b)

Figure 3: Modularization of community structure. (a) Modularity for online social support network. (b) Modularity for online social discussion network. 
athletes' online social networks. A relatively stable community structure is very important for the physical and mental development of athletes.

\section{Conclusion}

In summary, the network density of the athletes' online social support network is greater than that of the online social discussion network, and specifically among the subnetworks, the practical online support network is the most dense and the love and marriage online discussion network is the least dense. This indicates that in recent years professional athletes have been gradually increasing their online practical support claims in the course of their daily lives and training. Athletes also show higher social online support, indicating that, in the new era and environment, new athletes still attach more importance to social interaction, and they are actively expanding their life circle.

The proportion of the relational composition of athletes' online social support networks has also changed in a more interesting way. While the proportion of teammates and family members remains high overall, some studies have shown that the proportion of social support provided by family members has declined significantly, while the social support provided by classmates or friends even surpasses that of family members [27], a feature that is particularly evident when it comes to social online support. Of course, it is still important not to overlook the strong social support power of teammates, which is unmatched by any other social role.

In terms of strong and weak relationships, athletes' online social support networks still show a strong tendency towards "strong relationships," but compared to online social discussion networks, weak relationships are slowly gaining ground, especially in social online support, where the proportion of weak relationships reaches almost $40 \%$. This is mainly due to the fact that classmates or friends play a greater role in this area, while team managers have the lowest percentage, which is also related to the authority of the managers and the fact that athletes are more distant from coaches and instructors and have to turn to their teammates or classmates for social support.

\section{Data Availability}

The original data used in this study are the questionnaire data obtained from the survey. The original data used to support the findings of this study are available from the corresponding author upon request.

\section{Conflicts of Interest}

The authors declare that they have no conflicts of interest.

\section{Acknowledgments}

This work was supported in part by the National Natural Science Foundation of China under Grant nos. 61440036 and 61040029 and the National Social Science Foundation of China under Grant no. 16ATY002.

\section{References}

[1] J. Ding and H. Qian, "A study on the multi-level relationship between the overall social network of Chinese professional athletes," in Proceedings of the 2015 Abstract of papers of the Tenth National Sports Science Congress, pp. 1217-1218, China Sports Science Society, Hangzhou, Jiangsu, January 2015.

[2] H. Qian and X. Zhang, "Effects of personal characteristics of Chinese professional athletes on the structure of social support network," Journal of Shanghai Institute of Physical Education, vol. 38, no. 6, pp. 59-63, 2014.

[3] H. Qian, X. Yang, J. Ding et al., "Analysis of social network characteristics of Chinese professional athletes," Journal of Wuhan Institute of Physical Education, vol. 50, no. 7, pp. 77-83, 2016.

[4] H. Qian and J. Ding, "Analysis of the overall social network characteristics of Chinese professional athletes," in Proceedings of the Compendium of Abstracts of the 10th National Sports Science Conference 2015, pp. 811-813, Chinese Society of Sports Science, Hangzhou, Jiangsu, September 2015.

[5] X. Zhang and H. Qian, "Research on structural characteristics of job search network of Chinese professional athletes-based on sample survey of professional athletes in Shaanxi province," Journal of Wuhan Institute of Physical Education, vol. 52, no. 3, pp. 17-23, 2018.

[6] Z. Xiong, A Study on Community Discovery Technology and its Application in Online Social Network, Central South University, Changsha, China, 2012.

[7] C. Xiao, Analysis and Prediction of User's Behavior in Online Social Network, University of Electronic Science and Technology of China, Chengdu, China, 2013.

[8] Z. Li, A Study on the Measurement and Law of Information Dissemination of Node Information in Social Network, Harbin Institute of Technology, Harbin, China, 2015.

[9] Y. Su, A Study on the Communication Model and Intervention of Public Opinion in Online Social Network, Yanshan University, Qinhuangdao, China, 2018.

[10] L. Wang and X. Cheng, "Dynamic community discovery and evolution of online social network," Journal of Computer Science, vol. 38, no. 2, pp. 219-237, 2014.

[11] Y. Chen, Z. Li, X. Liang et al., "A review of online social network rumor detection," Chinese Journal of Computers, vol. 41, no. 7, pp. 1648-1677, 2017.

[12] Li Qian, B. Liu, and Y. Zhao, "Construction of statistical metadata in the big data environment," Statistics and Information Forum, vol. 35, no. 03, pp. 14-20, 2020.

[13] R. Zhang, L.-X. Liu, X.-B. Tang, and B.-R. Zhang, "Research on the prediction of commodity retail price index based on web search data in the context of big data," Statistics and Information Forum, vol. 35, no. 11, pp. 49-56, 2020.

[14] Z. Pang and M. Zeng, "Has internet use affected youth subjective well-being? -an analysis of CGSS data from 2010-2015," Journal of Xi'an University of Finance and Economics, vol. 33, no. 03, pp. 71-77, 2020.

[15] H. Li and Y. Chen, "Research on the generation and dissemination mechanism of network public opinion-based on the perspective of big data social network analysis," Age of Media Contemporary Communications, vol. 186, no. 1, pp. 24-25, 2016.

[16] X. Wang, "Data science and social networks: big data, small world," Science and Society, vol. 1, no. 1, pp. 27-35, 2014.

[17] M. Jia, H. Xu, J. Wang et al., Handling Big Data of Online Social Networks on a Small Machine, Springer International Publishing, New York, NY, USA, 2015. 
[18] Y. Tian, Y. Lin, and L. Gong, "Research on the influence of Internet culture on campus culture in the Internet era--analysis based on questionnaire survey of teachers and students in some universities in Shaanxi," Statistics and Information Forum, vol. 35, no. 07 , pp. 122-128, 2020.

[19] J. Ding, L. Xu, H. Qian et al., "A study on the multi-level relationship of the overall social network of Chinese professional athletes," Journal of Xi'an Institute of Physical Education Nol, vol. 4, pp. 405-409, 2016.

[20] J. Ding and H. Qian, "A central analysis of the overall social network of professional athletes in China," A Journal of Xi'an University of Technology, vol. 36, no. 5, pp. 408-413, 2016.

[21] Y. Qian, Online Social Networking Analysis, Electronic Industry Press, Beijing, China, 2014.

[22] Y. Zhao and J. Luo, "How to measure social capital: a review of empirical research," Social Sciences Abroad, no. 2, pp. 18-24, 2005.

[23] X. Jin, Y. Ren, and H. du, Change of Social Network and Conceptual Behavior of Migrant Workers, Social Sciences Press, Beijing, China, 2014.

[24] J. S. A. W. Carolyn, "AP primer: logit models for social networks," Social Networks, vol. 21, no. 1, pp. 37-66, 1999.

[25] Z. Li and J. Luo, "A view on local management theory from the perspective of social network," Journal of Management, vol. 8, no. 12, pp. 1737-1747, 2011.

[26] Z. Li and J. Luo, "The social behavior and relational network characteristics of Chinese-viewpoint of a social network," Social Science Front, vol. 199, no. 1, pp. 159-164, 2012.

[27] J. Luo and F. Zeng, "A study on organization based on complex system perspective," Foreign Economics and Management, vol. 41, no. 12, pp. 112-134, 2019.

[28] J. Liu, "Practical Guide to Integrated Network Analysis UCINET Software, GE Zhi Publishing House, " Shanghai, China, 2014.

[29] K. Xu, S. Zhang, H. Chen et al., "Measurement and analysis of online social network," Chinese Journal of Computer, vol. 37, no. 1, pp. 165-188, 2014.

[30] Y. Ren, S. Li, H. Du et al., "An analysis of the social network structure of migrant workers," A Journal of Xi'an Jiaotong University (Social Sciences Edition), vol. 91, no. 5, pp. 44-51, 2008.

[31] Q. Huang, S. F. Fung, B. Liu et al., "Modeling for professional athletes' social networks based on statistical machine learning," Advanced Data Mining Methods for Social Computing, vol. 8, 2019.

[32] J. Liu, “A study on social network model," Sociological Studies, vol. 1, pp. 1-12, 2004.

[33] J. Liu, A Study on Social Network Extraction Technology for Text-Oriented Information and its Application, University of Defence Science and Technology, Changsha, China, 2009.

[34] Y. Wang, Field, T. Li et al., "Visual analysis of shipping recruitment information based on Gephi," Big Data, vol. 4, no. 3, pp. 81-91, 2018.

[35] S. Hussain, L. Muhammad, and Y. Atomsa, "Mining social media and DBpedia data using Gephi and R," Journal of Applied Computer Science \& Mathematics, vol. 12, no. 25, pp. 14-20, 2018.

[36] Y. Guan, Y. Xiang, and C. Kang, "Research and application of visual analysis method based on Gephi," Telecommunications Science, vol. 29, no. S1, pp. 112-119, 2013.

[37] F. Xiong, X. Wang, S. Pan, H. Yang, H. Wang, and C. Zhang, "Social recommendation with evolutionary opinion dynamics," IEEE Transactions on Systems, Man, and Cybernetics: Systems, vol. 50, no. 10, pp. 3804-3816, 2020.
[38] Y. Hu, F. Xiong, S. Pan, X. Xiong, L. Wang, and H. Chen, "Bayesian personalized ranking based on multiple-layer neighborhoods," Information Sciences, vol. 542, pp. 156-176, 2021.

[39] F. Xiong, W. Shen, H. Chen, S. Pan, X. Wang, and Z. Yan, "Exploiting implicit influence from information propagation for social recommendation," IEEE Transactions on Cybernetics, vol. 50, no. 10, pp. 4186-4199, 2020.

[40] S. L. Deng, B. Wang, B. Wu et al., "Modeling and validation of complex network association partitioning based on information entropy," Computer Research and Development, vol. 49, no. 4, pp. 725-734, 2012. 